in practice psychiatry is still a shortage speciality. Trainees can therefore be encouraged to apply for registrar posts as soon as they pass their Part I; thus it is anticipated that the length of time at SHO level will be relatively short especially for "high flyers".

The increased supervision of training both for SHOs and registrars required by Achieving $a$ Balance is surely beneficial to all trainees. Career counselling for "stuck doctors" is obviously very important but is currently seldom carried out in a systematic way. Achieving a Balance requirements clearly remedy this. Regular and formal review of registrars' progress by a regional based committee is also surely to be welcomed.

Thus although the requirements of Achieving $a$ Balance involves the local scheme organisers, regional advisers, clinical tutors as well as College convenors and their teams with extra work, rotational schemes can be devised which benefit trainees. In such cases "controversy, ill-feeling and loss of morale" should not occur.

Adviser in Postgraduate Psychiatric Education

University of Wales College of Medicine

Cardiff CF4 $4 X N$

\section{Discharge summaries}

\section{DeAR Sirs}

With reference to the article in the series 'Audit in practice' entitled 'Audit of psychiatric discharge summaries' (Psychiatric Bulletin, October 1990, 14, 618-620). I was somewhat concerned to note that there did not appear to be any attention paid to the fact that many general practitioners either read the discharge summaries to the patient, hand the summary to the patient so that he or she can read it, or leave the patient in the surgery with the summary conveniently placed in front of him/her while the GP attends to other matters.

While I agree that a good summary is important for the psychiatric notes, I would feel that the best method of producing a summary for the GP should consist of the name and address of the patient, a diagnosis not exceeding six words, and the current medication and whether or not there is follow-up from the psychiatric service and in what form this would be.

Possibly, given the fact that at Highcroft Hospital there are 23 psychiatrists, the average contact with GPs is so low that they have not experienced these matters.

\section{Burnley General Hospital \\ Burnley, Lancs BB10 2PQ}

Michael Launer

DeAR SirS

As we cited in our Bulletin report 'Audit of psychiatric discharge summaries', we have conducted a questionnaire study of the 234 general practitioners who refer patients to Highcroft Hospital in order to determine their preferences for format of a discharge communication from psychiatric hospital (Craddock \& Craddock, 1989). We asked general practitioners to choose their preferred summary from three specimen summaries and $208(89 \%)$ general practitioners responded. The briefest summary (very similar to Dr Launer's suggestion) was chosen by only $8 \%$ of respondents; $26 \%$ opted for a full and detailed summary filling $2 \frac{1}{4}$ sides of A4 typescript while the majority $(66 \%)$ preferred a summary of intermediate detail (with a length of one side of A4 typescript). We used the same methodology to determine which specimen summary the 23 psychiatrists at Highcroft Hospital preferred to have filled in the case notes as a record of the admission: $74 \%$ opted for the detailed summary and $26 \%$ for the summary of intermediate detail. There was a significant difference $(P<0.001)$ between the preferences of general practitioners and psychiatrists and we concluded that a single summary cannot adequately meet the needs of both psychiatrists and general practitioners.

We suggest that general practitioners are sent a summary on one side of A4 typescript which contains details specifically pertinent to the general practitioner's future management of the case (which will include many, but not all, of the 23 items we list in our Bulletin report). We believe that the general practitioner should also be sent a copy of the detailed hospital summary (which may be discarded if not wanted). Such a scheme would satisfy the preferences of $92 \%$ of the general practitioners we surveyed.

Nick CRADDOCK

Queen Elizabeth Hospital

Bridget CRADDOCK Birmingham B15 2TH

\section{Reference}

Craddock, N. \& Craddock, B. (1989) Psychiatric discharge summaries: differing requirements of psychiatrists and general practitioners. British Medical Journal, 299, 1382.

\section{A form of drug audit in mental handicap}

\section{Dear Sirs}

The drug treatment of mentally handicapped patients in hospital and community is complicated by issues which do not arise as frequently in general psychiatric practice. In mentally handicapped people there is, first, the question of how their level of understanding affects their capacity to consent to treatment. Second, psychiatric diagnoses are often less clear cut. Third, abnormal brain structure and function may affect the response to drugs. Fourth, carers 